\title{
Formal Characteristics of Thematic Apperception Test Narratives of Adult Patients with an Autism Spectrum Disorder. A Preliminary Study
}

\author{
Elisabeth Eurelings-Bontekoe ${ }^{1}$, Kirsten Zwinkels ${ }^{1}$, Hanneke Schaap-Jonker ${ }^{2,3}$, \\ Moshen Edrisi ${ }^{4}$ \\ ${ }^{1}$ Institute for Psychological Research, Clinical Psychology Unit, University of Leiden, Leiden, The Netherlands; \\ ${ }^{2}$ Faculty of Theology and Religious Studies, University of Groningen, Groningen, The Netherlands; \\ ${ }^{3}$ Dimence Institute of Mental Health, Zwolle, The Netherlands; \\ ${ }^{4}$ GGZ Divers, Leiden, The Netherlands. \\ Email: eureling@fsw.leidenuniv.nl \\ Received July $19^{\text {th }}, 2011$; revised August $23^{\text {rd }}, 2011$; accepted September $26^{\text {th }}, 2011$.
}

\begin{abstract}
This study explored the usefulness of a Thematic Apperception Test (TAT, Murray, 1943) narrative scoring system to detect signs of autistic information processing. A total of 27 patients with an Autism Spectrum Disorder were compared to a control group $(\mathrm{N}=67)$ of patients with other psychiatric disorders. As an external indirect measure of social functioning two dimensions of the Social Cognition and Object Relation Scale (SCORS; Westen,1985) were used: Capacity for Emotional and Moral Investment in Relationships and Understanding of Social Causality. TAT narratives were rated on the prevalence of eight phenomena, as proposed by Edrisi and Eurelings-Bontekoe (2009). Autistic patients showed a lower level of social insight and were particularly characterized by high scores on Weak Central Coherence, Jumping to Conclusions and Difficulty with describing/ interpreting movements. Results provide preliminary support for the usefulness of the TAT scoring system, but need to be replicated using larger samples and blind scoring.
\end{abstract}

Keywords: Autism, Thematic Apperception Test, Social Cognition, Adults

\section{Introduction}

Autism Spectrum Disorders (ASDs) are neurodevelopmental disorders, characterized by a triad of symptoms: limited reciprocal social interactions, disordered verbal and nonverbal communication, and restricted, repetitive behaviors or circumscribed interests (Tager-Flusberg \& Caronna, 2007). The DSMIV Pervasive Developmental Disorders section includes five different disorders: Autistic Disorder (AD), Rett's disorder (RD), Childhood Disintegrative Disorder (CDD), Asperger's Syndrome (AS), and Pervasive Developmental Disorder Not Otherwise Specified (PDD-NOS) (American Psychiatric Association, 2000). Patients with Autism, AS and PDD-NOS share the feature of limited reciprocal social interactions. In addition to this feature, patients with Autism show disordered verbal and nonverbal communication and restricted, repetitive behaviors or circumscribed interests. Patients with AS suffer from limited reciprocal social interactions, and show restricted, repetitive behaviors or circumscribed interests, but no deficits in verbal or nonverbal communication. Finally, patients are diagnosed with PDD-NOS if they suffer from limited reciprocal social interactions combined with either disordered verbal or non verbal communication or restricted repetitive behaviors. In addition to the DSM-IV-TR (APA, 2000) criteria, three functional criteria may be described: impaired executive functioning (Ozonoff, South, \& Provencal, 2005), weak central coherence (Happé \& Frith, 2006), and impaired theory of mind (BaronCohen, 2000; Tager-Flusberg, 2007). According to Minshew, Sweeney, and Luna (2002), information processing is disordered in patients with ASDs. Problems occur especially in complex information processing, involving the processes that pose the highest information processing demands (Beaumont \& Newcombe, 2006).

\section{Assessment Using the Thematic Apperception Test}

The Thematic Apperception Test (TAT; Murray, 1943) was originally designed to measure normal dimensions of personality in the general population (Langan-Fox \& Grant, 2006). Westen (1985, 1991a, 1991b) developed the Social Cognition and Object Relations Scale (SCORS) to score TAT narratives.

Social cognition is defined by the thought processes involved in understanding of behavior and motives of other people, whereas object relations pertain to the individual's thoughts and feelings about people (Westen, 1991b). From this social cognition perspective, the TAT is an excellent instrument, because it asks subjects to draw on their internal object representations to construct characters and interaction in response to an ambiguous interpersonal situation (Cramer, 1999). Originally, Westen (1985) distinguished four dimensions within the SCORS: 1) Complexity of representations of people, (COM) assesses the extent to which the subject clearly differentiates the perspectives of self and others; 2) Affect tone of relationship paradigms (ATT), assesses affective quality of representations of people and relationships; 3) Capacity for emotional investment in relationships and moral standards (EI), assesses the extent to which inner representations of relationships reflect an egocentric attitude; and 4) Understanding of social causality (SOC), assesses a patients' capacity to understand causal relationships in social interactions. In diagnosing Autism the dimensions EI and SOC seem to be most informative. 
The TAT has mainly been used in studies among children with ASD as opposed to adults and quantitative data concerning the coherence of narratives of adult ASD patients are virtually lacking (Beaumont \& Newcombe, 2006). The idea of using the TAT in the assessment of Autism among adults is quite novel and unconventional. Indeed, to the best of our knowledge, there is only one study that addressed the coherence of narratives of adult ASD patients (Beaumont \& Newcombe, 2006). These authors compared the TAT narratives of 20 patients with High Functioning Autism (HFA)-AS with those of 20 controls regarding the mean proportion of mental state words, of mental state causal statements and of action causal statements. The two groups differed only with respect to the proportion of mental state causal statements, in such a way, that control narratives included a significant greater proportion of mental state causal statements than the narratives of ASD patients, implying that, although HFA-AS patients tend to use mental state words to a similar extent as controls, they are less inclined to provide explanations for characters mental state than controls (Beaumont $\&$ Newcombe, 2006).

Recently, Edrisi and Eurelings-Bontekoe (2009, 2011) suggested the usefulness of the TAT in detecting signs of ASD. On the basis of several years of clinical experience, they have described eight phenomena that are considered typical for patients with ASD.

\section{Aims of the Study and Hypotheses}

The aim of this study was to explore the validity of the TAT ASD scoring system as described by Edrisi and EurelingsBontekoe (2009). This was done in two ways. First, we studied the association between the percentage of ASD phenomena in TAT stories and the SCORS dimensions EI and SOC. Next, we compared patients with ASDs and psychiatric controls regarding mean scores on EI and SOC and the percentage of ASD phenomena in TAT narratives. We hypothesized that the correlations between the percentage of ASD phenomena in TAT narratives and the scores on the SCORS dimensions EI and SOC would all be negative. Furthermore, we hypothesized that patients with ASD would score lower on EI and SOC and would show a higher percentage of ASD phenomena in TAT narratives than the psychiatric controls.

\section{Material and Methods}

\section{Participants}

Twenty seven patients with a diagnosis in the autistic spectrum and in treatment (either ambulatory or in some living arrangement) in a specialized mental health care setting participated in the study. The group consisted of six females $(22.2 \%)$ and 19 males $(70.4 \%)$. Gender was unknown for two patients (7.4\%). Age ranged between 19 and 54 years, with a mean age of 30.6 years, $(\mathrm{SD}=9.71)$. Four patients $(14.8 \%)$ were diagnosed with Autism, six patients $(22.2 \%)$ were diagnosed with AS and 17 (62.9\%) with PDD-NOS.

The control group was matched on gender. Control TAT data were selected from data collected by Eurelings-Bontekoe among a large and heterogeneous sample of psychiatric patients (Eurelings-Bontekoe, Luyten, \& Snellen, 2009). The control group $(\mathrm{N}=67)$ consisted of 19 females $(28.4 \%)$ and 48 males (71.6\%). The average age of this group was 39.4 years $(\mathrm{SD}=$ 10.51). Age ranged between 22 and 60 years. The exclusion criterion for this group was an ASD diagnosis.

\section{Measures}

The Social Cognition and Object Relation Scale (SCORS; Westen, 1985, 1991a, 1991b) was used as an external indirect measure of social functioning. In this study the following two dimensions as originally described by Westen (1985) were rated: Capacity for Emotional and Moral Investment in Relationships (EI), as a measure of affective social functioning and Understanding of Social Causality (SOC), as a measure of cognitive social functioning. Low scores on EI reflect an egocentric and selfish attitude, high scores reflect the capacity to establish and maintain reciprocal social interactions. SOC assesses a patient's capacity to understand causal relationships in social interactions. Low scores reflect illogical causal relationships, high scores reflect understanding of causal relationships in terms of both inner motives and external circumstances. Each dimension is scored on a five-point rating scale, with higher scores on EI and SOC representing higher levels of social functioning. Studies have provided considerable evidence for the convergent and discriminant validity of the SCORS (Ackerman, Hilsenroth, Clemence, Weatherill, \& Fowler, 2001, Cramer, 1999; Peters, Hilsenroth, Eudell-Simmons, Blagys, \& Handler, 2006; Porcerelli, Shahar, Blatt, Ford, \& Greenlee, 2006; for a review, see Huprich \& Greenberg, 2003). Interrater reliability of the scoring of the TAT protocols of the ASD patients (ICC $(2,2))$ was .88 for EI and .96 for SOC (both excellent). ICC $(2,1)$ were respectively .79 and .92 (excellent; see Table 1). The TAT protocols of the control group had already been scored on the SCORS variables EI and SOC with good to excellent interrater reliability (Eurelings-Bontekoe et al., 2009).

The second measure used in this study was the rating of ASD phenomena in TAT narratives. Based on clinical experience, Edrisi and Eurelings-Bontekoe (2009) defined and described eight phenomena that they consider typical formal characteristics of TAT protocols of patients with ASD:

1) Weak Central Coherence, i.e. trying to understand the meaning of the picture by concentrating on details, including irrelevant ones, instead of utilizing the whole context. Example:

Table 1.

ICC two way random effect model, single $(2,1)$ and average measures (2.2) for SCORS dimensions EI and SOC and for ASD phenomena.

\begin{tabular}{ccc}
\hline Variables & Single measures & Average measures \\
\hline SCORS Emotional Investment & .79 & .88 \\
SCORS Social Causality & .92 & .96 \\
Weak Central Coherence & .93 & .96 \\
Ruminating & .85 & .92 \\
Weak Theory of Mind & .91 & .95 \\
Jumping to Conclusions & .61 & .76 \\
Multiple Scenario's & .98 & .99 \\
No Social Connectedness & .85 & .92 \\
No Parallel Processes & .47 & .64 \\
$\begin{array}{c}\text { Problems with describing/ } \\
\text { interpreting movements }\end{array}$ & .75 & .86 \\
\hline
\end{tabular}


"I see a boy, looking at a violin with a bow. The violin lies on the table on top of a piece of paper or a piece of cloth. I can not see whether there is music on it".

2) Ruminating. The patient does not know what an object or person in the picture stands for and starts ruminating about it. Patients can not turn their attention away from certain associations. Example: "The room is a little bit dark, I think the light comes from outside. However, the light may also come from the lamp, because on the left side of the room there is no light from the hallway and the right side of the room is a little lighter. And at some places you can see the shadows the lamp casts".

3) Underdeveloped Theory of Mind, i.e. the patient is unable to theorize about what the persons in the picture could feel and think. Emotions are derived from external cues like eyes, mouth, and posture. Examples: "He is depressed, because of his mouth"; "he is thinking, because his left eyebrow is raised higher than his right". If the patient cannot see a person's face, he is unable to tell what this person could be feeling or thinking: "I cannot tell what the man is feeling, because I only see his back".

4) Jumping to Conclusions, i.e. the patient makes illogical inferences and "jumps to conclusions". For example: "This woman has her eyes closed, so she is practising yoga". Or: "This is a single bed, so they cannot be a couple". Or: "Their hands look similar, so they are brother and sister".

5) Describing multiple scenario's. The patient describes several potential scenarios to explain what could be going on. Example: "He is sad, because something happened to the violin, or he is angry because it is broken, or he is anxious because he is unable to play the violin".

6) Lack of social connectedness. Persons on the picture do not interact or are not related to each other. Sometimes they are described as being "pasted" into the picture. Example: "Well, there are three figures, three people. Let's see. This lady in front is staring in the distance. They are not looking at the public, neither is the horse. This is strange ... what happens is ... it is very incoherent. Those people are not related to each other, they are not connected. It is all individual. Yes, there is no interaction, ... At the end, he goes on working, she is going home to eat and she will go on studying".

7) Inability to describe parallel processes, i.e. the patient is unable to describe events simultaneously, but instead tends to describe the events consecutively. Example: "After they have stopped hugging they will eat".

8) Problems with describing and interpreting movements spontaneously. The patient has trouble describing and interpreting movements in frozen images. Movements are described on the basis of posture or direction in which the person looks. Example: "Her shoulder is bent towards the room, therefore she is entering the room". Or "The woman looks to the right side of the picture, therefore she is walking into that direction". Difficulties with the interpretation of movement may very well be clarified by the story of a patient with PDD-NOS who described this inability as follows: "In many pictures it seems as if the picture has been taken while people on the picture are moving. Since you do not see anything else you cannot tell whether a person is for instance leaving or entering a room. Closed eyes are also difficult. It is possible that at the moment the picture was taken the man accidentally blinked his eyes, but it is equally possible that his eyes were already closed for a longer time, yes, than he may be unconscious, that is more dramatic".

Concerning the ASS phenomena, ICC $(2,1)$ was excellent for all phenomena except for the phenomena Jumping to Conclusions and of No Parallel Processes, for which ICC's $(2,1)$ were respectively good and fair. However ICC's average measures
$(2,2)$ were good for No Parallel Processes and excellent for the rest of the ASD phenomena (see Table 1).

\section{Procedure}

The participants in the ASD group $(\mathrm{N}=27)$ who had agreed to participate in the study were paid a house visit by one of several (eight) investigators. Investigators were psychologists, test assistants, or nurses, who were qualified or instructed to take TAT-interviews. Interviews took about one hour. The TAT was administered using standard guidelines (Conklin \& Westen, 2001), asking the patient to describe what happens, what led up to the situation, what the outcome would be, and what the characters would be thinking and feeling. The following six TAT cards were administered in the same order to all ASD patients: 1, 2, 5, $6 \mathrm{BM}, 13 \mathrm{MF}$, and $18 \mathrm{BM}$. All narratives were recorded on audiotape and transcribed verbatim. Sixty seven control cases were selected. TAT pictures used in this group were 1, 2, $13 \mathrm{MF}, 4,3 \mathrm{BM}, 7 \mathrm{GF}$.

The TAT protocols of the control group had already been scored on the SCORS variables EI and SOC. The second author scored the protocols of the ASD patients for EI and SOC and scored all protocols, including those of the psychiatric controls for the occurrence of each of the eight ASD phenomena, after having been trained by the first author. The ratings for the two SCORS dimensions were averaged across the six TAT cards yielding a single mean score on each dimension for each patient.

For each patient and each protocol, the frequency of occurrence of the above-mentioned ASD phenomena was counted. Since the amount of recognizable phenomena in each story is dependent upon the length of the protocol, protocol length was corrected for by dividing the frequency of each ASD phenomenon per protocol by the number of words in each protocol and by multiplying this number by 100 yielding a percentage score for each ASD phenomenon per protocol. These percentage scores of ASD phenomena were used in the MANCOVA and Discriminant Analysis. To be able to establish interrater reliability for the present study, the third author independently scored a random selection of 20 ASD protocols for the SCORS variables SOC and EI and for the ASD phenomena. The Intraclass Correlation Coefficient, two way random effect model (ICC; Shrout \& Fleiss, 1979) was used to calculate interrater reliability. ICCs are considered to be excellent if greater than .74 , good if ranging from .60 to .74 , fair, if ranging from. 40 to .59 and poor if under .40 (Cicchetti, 1994).

\section{Analyses}

Groups were compared regarding gender and age using Chisquare statistics and t-test for independent samples respecttively. In order to investigate whether the TAT ASD phenomena were associated with social functioning as operationalized by the two SCORS variables SOC and EI, we calculated Pearson product-moment correlations between these SCORS variables and the ASD phenomena.

MANCOVA was used in order to study the differences between ASD patients and psychiatric controls regarding mean scores on EI and SOC, mean percentages of ASD phenomena and the number of words per protocol with age as a covariate. Effect sizes were calculated in terms of partial eta squared ( $р 2$ ). According to conventional criteria (Cohen, 1988) a $\eta p 2$ of .01 is small; .06 moderate; .14 large. Cohen's d's were calculated as an Effect Size (ES) measure of differences between groups (Cohen, 1977). According to conventional criteria, d 
$\leq .20$ is considered a small $\mathrm{ES} ; \mathrm{d}=.50$ a medium $\mathrm{ES} ;$ an $\mathrm{d} \geq .80$ a large ES.

Finally, a canonical Discriminant Analysis (method stepwise) was used to investigate the power of the ASD TAT phenomena, to discriminate between the ASD group and the psychiatric controls.

\section{Results}

\section{Influence of Background Variables}

Gender was evenly distributed across the two groups $\left(\chi^{2}\right.$ $=.18 ; \mathrm{df}=1, \mathrm{p}=.68)$. The groups did however differ from each other regarding age $(\mathrm{t}(92)=-3.75, \mathrm{p}<.05)$. As a consequence, age was taken into account as a covariate.

\section{Correlations between SCORS Variables and ASD Phenomena}

Table 2 shows that all but two ASD phenomena, Multiple Scenario's and Inability to describe parallel processes, were negatively associated with the two SCORS variables. Difficulties with describing/interpreting movements was negatively correlated with EI only.

\section{Differences between the Groups on ASD Phenomena}

MANCOVA was used to compare groups regarding mean scores on the SCORS variables EI and SOC, the ASD phenomena, and the mean number of words per protocol. Age was used as covariate.

There was a significant main effect for group $(\mathrm{F}(11,93)=$ $18.46, \mathrm{p}<.001$ with a huge ES $(\eta \mathrm{p} 2=.72)$; ASD patients scored significantly lower than psychiatric controls with large ESs (with the exception of SOC, where a medium ES was found) on the following variables: $\operatorname{EI}(F(1,93)=23.35$, $p$ $<.001, \mathrm{~d}=.1 .16 ; \mathrm{M}=1.34, \mathrm{SD}=.26$ and $\mathrm{M}=1.68, \mathrm{SD}=.31$ respectively), $\operatorname{SOC}(\mathrm{F}(1,93)=7.28, \mathrm{p}<.01, \mathrm{~d}=.53 ; \mathrm{M}=1.59$, $\mathrm{SD}=.30$ and $\mathrm{M}=1.79, \mathrm{SD}=.41$ respectively), Weak Central Coherence $(\mathrm{F}(1,93)=46.20, \mathrm{p}<.001 ; \mathrm{d}=1.68 ; \mathrm{M}=.24$, SD $=.19$ and $\mathrm{M}=.03, \mathrm{SD}=.09$ respectively $)$, Ruminating $(\mathrm{F}(1,93)$ $=12.74, \mathrm{p}<.01 ; \mathrm{d}=.79 ; \mathrm{M}=.25, \mathrm{SD}=.18$ and $\mathrm{M}=.12$, $\mathrm{SD}=.16$ respectively), Underdeveloped Theory of Mind $(\mathrm{F}(1$, $93)=23.41, \mathrm{p}<.001 ; \mathrm{d}=1.08 ; \mathrm{M}=.59, \mathrm{SD}=.42$ and $\mathrm{M}=.18$, $\mathrm{SD}=.37$ respectively), Jumping to Conclusions $(\mathrm{F}(1,93)=$
$17.67, \mathrm{p}<.001 ; \mathrm{d}=1.03 ; \mathrm{M}=.83, \mathrm{SD}=.78$ and $\mathrm{M}=.35, \mathrm{SD}$ $=.26$ respectively), No social connectedness $(\mathrm{F}(1,93)=17.35$, $\mathrm{p}<.001, \mathrm{~d}=1.07 ; \mathrm{M}=.33, \mathrm{SD}=.28$ and $\mathrm{M}=.11, \mathrm{SD}=.17$ respectively), and Problems with describing/interpreting movements, $(\mathrm{F}(1,93)=27.20, \mathrm{p}<.001 ; \mathrm{d}=1.40, \mathrm{M}=.10, \mathrm{SD}=.12$ and $\mathrm{M}=.005, \mathrm{SD}=.03$ respectively). In addition, the mean number of words in protocols of ASD patients was significantly higher than that in protocols of psychiatric controls $(\mathrm{F}(1,93)=$ $22.51, \mathrm{p}<.001 ; \mathrm{d}=1.08), \mathrm{M}=1123.44, \mathrm{SD}=674.51$ and $\mathrm{M}=$ $649,14, \mathrm{SD}=305.90$ respectively).

No significant differences were found for Multiple Scenario's $(\mathrm{F}(1,93)=2.44$, ns $)$, and Inability to see parallel processes, $(F(1,93)=.45, \mathrm{~ns})$.

\section{Which TAT Phenomena Discriminate Best between the Two Groups?}

Finally, a Discriminant Analysis, method stepwise, was used to discriminate the ASD group from the control group on the basis of the ASD phenomena.

The analysis yielded one discriminant function with an Eigenvalue of 1.33 (Wilks $\lambda=.43 ; \chi^{2}=75.77$; $\mathrm{df}=5 ; \mathrm{p}<.001$ ). Canonical correlation was .76. For results see Table 3. This function is dominated by Lack of Central Coherence (.67; Table 3(a)). Table 3(b) shows the correlations of the variables with the discriminant function. Table 3(c) displays the function at group centroid, showing that this discriminant function separates the ASD group from the control group. Therefore, cases in the ASD group were particularly characterized by high scores on Lack of Central Coherence, Problems with describing/interpreting movements and Jumping to Conclusions as opposed to cases in the control group, which scored low on these variables. On the basis of this discriminant function $91.5 \%$ of cases could be classified correctly (see Table 3(d)). Sensitivity was $81 \%$ and specificity was $96 \%$. These figures imply that nineteen out of hundred persons with ASD receive a false negative diagnosis, whereas four out of hundred persons without ASD receive a false positive diagnosis. Positive Predictive Power (PPP), or the probability that a person with scores typical for the ASD group actually has an ASD, was .88. Negative Predictive Power (NPP), or the probability that a person without scores typical for the group with ASD actually has no ASD, was .93. It should be kept in mind that, unlike Sensitivity and

Table 2.

Pearsons correlations between the SCORS variables and the ASD TAT phenomena $(N=93)$.

\begin{tabular}{ccc}
\hline ASD Phenomena & SCORS Emotional Investment & SCORS Understanding Social Causality \\
\hline Weak central coherence & $-.40^{* *}$ & $-.32^{* *}$ \\
Ruminating & $-.29^{* *}$ & $-.25^{*}$ \\
Weak theory of mind & $-.52^{* *}$ & $-.48^{* *}$ \\
Jumping to conclusions & $-.34^{* *}$ & $-.24^{*}$ \\
Multiple scenario's & -.08 & -.11 \\
No social connectedness & $-.44^{* *}$ & $-.34^{* *}$ \\
No parallel processes & -.04 & -.01 \\
Problems with describing/interpreting movements & $-.27^{* *}$ & -.18 \\
Total ASD phenomena & $-.58^{* *}$ & $-.49^{* *}$
\end{tabular}

Note: $* *$ Correlation is significant at the .01 level (2-tailed). ${ }^{*}$ Correlation is significant at the .05 level (2-tailed). 
Table 3.

(a) Standardized canonical discriminant function coefficients; (b) Pooled within-groups correlations between discriminating variables and standardized canonical discriminant function; (c) Function at group centroids; (d) Classification of results for predicted group membership.

(a)

\begin{tabular}{cc}
\hline Variables & Function 1 \\
\hline Weak central coherence & .67 \\
Multiple scenario's & .41 \\
Problems with describing movement & .41 \\
Jumping to conclusions & .38 \\
Ruminating & .37 \\
\hline
\end{tabular}

(b)

\begin{tabular}{cc}
\hline Variables & Function 1 \\
\hline Weak central coherence & .66 \\
Problems with describing movements & .54 \\
Jumping to conclusions & .41 \\
Ruminating & .30 \\
Multiple scenario's & .16 \\
\hline
\end{tabular}

(c)

\begin{tabular}{ccc}
\hline Group & Function 1 \\
\hline ASD group & 1.80 \\
Control group & -.73 \\
\hline
\end{tabular}

(d)

\begin{tabular}{|c|c|c|c|c|c|c|c|}
\hline & & \multicolumn{6}{|c|}{ Predicted Group Membership } \\
\hline & & \multicolumn{2}{|c|}{ ASD group } & \multicolumn{2}{|c|}{ Control Group } & \multicolumn{2}{|c|}{ Total } \\
\hline & & Count & Percentage & Count & Percentage & Count & Percentage \\
\hline \multirow[t]{2}{*}{ Original Group } & $\mathrm{ASD}$ & 22 & 81.5 & 5 & 18.5 & 27 & 100.0 \\
\hline & Control group & 3 & 4.5 & 64 & 95.5 & 67 & 100.0 \\
\hline
\end{tabular}

Note: $91.5 \%$ of original grouped cases correctly classified.

Specificity, PPP and NPP are dependent upon the base rate of the condition in the population being tested. PPP decreases with decreasing prevalence. Likelihood ratio plus (LR+), expressed as sensitivity/1-specificity, was 20.25 , meaning that a positive result is 20 times as likely for those who have ASD as for those who do not suffer from ASD. Likelihood ratio minus (LR-), expressed as specificity/1-sensitivity, was 5.05 , indicating that low scores on the ASD phenomena are five times more likely to have come from a person who does not suffer from ASD than from a person who suffers from ASD. These figures imply that the odds for a false negative diagnosis are relatively greater than the odds for a false positive diagnosis.

\section{Discussion}

This study was aimed at exploring the usefulness of a TAT ASD scoring system, using ASD phenomena, as described by Edrisi and Eurelings-Bontekoe (2009). As expected, ASD patients differed significantly from psychiatric controls concern- ing all ASD phenomena, except for the phenomena Multiple Scenario's and Inability to Describe Parallel Processes. In addition, as compared to psychiatric controls ASD patients scored significantly lower on the SCORS dimensions EI and SOC, which is in line with a lower level of social-emotional insight among ASD patients as compared to psychiatric controls. As expected, mean scores on these two dimensions were negatively associated with the mean percentage of ASD phenomena in TAT narratives.

Also, the two groups differed significantly regarding the mean number of words of the TAT protocols, with patients with ASD using approximately twice as many words as psychiatric controls. The large number of words used by ASD patients may reflect their great effort to construct a story.

Discriminant analysis showed that cases in the ASD group were particularly characterized by high scores on Weak Central Coherence, Problems with describing/interpreting movements and Jumping to Conclusions as opposed to cases in the control group, which scored relatively low on these variables Weak 
central coherence of TAT narratives and jumping to conclusions reflect the inability of ASD patients to see "the Gestalt" of a picture and their limited insight into the fact that social interactions may be determined by a multiplicity of inner and outer causes. ASD patients tend to focus on one particular detail and compose the story around this detail. If this detail is irrelevant, this may result in an incomplete of completely incorrect interpretation of the social situation. Interestingly, the inability to describe and see movements spontaneously may parallel the results of Holaday, Moak and Shipley (2001), who compared Rorschach responses of 24 boys with AS, with that of 24 boys with behavioural or emotional disorders, and who found that the mean number of $\mathrm{M}$ (Movement) responses in the protocols of boys with AS was signifycantly lower as compared to that of the contrast group. A relatively low number of $\mathrm{M}$ responses refers to impaired reasoning and impaired imagination (Holaday et al., 2001).

Specificity (96\%) was somewhat higher than sensitivity $(81 \%)$. A relatively lower sensitivity than specificity increases the probability of a false negative diagnosis, as was also shown by the Likelihood ratio minus ( $\mathrm{LR}^{-}$). On the other hand, a high specificity has the advantage of decreasing the probability of a false positive diagnosis. In other words, the TAT scoring system has the risk of missing patients with ASS, but has a lower risk of falsely diagnosing patients with ASS, who in fact do not suffer from the condition. Therefore, although further assessment is always needed, this is especially so in case a patient is clinically suspected for ASD, even if the TAT narratives do not show the typical ASD phenomena.

\section{Limitations}

The results of this study need to be viewed in de context of several limitations.

First, the researcher scoring the narratives was aware of which group the stories belonged to. However, with the exception of Multiple Scenario's and No Parallel Processes, each of the ASD phenomena and the total mean percentage of ASD phenomena were negatively correlated with affective and cognitive aspects of social functioning as measured with the SCORS. Moreover, the TAT protocols from the ASS patients showed a significant lower level of emotional investment and understanding of social causality than those of the psychiatric controls. The TAT protocols of the 67 controls had already been scored on SCORS variables outside the context of this study. Furthermore the two groups did not differ from each other regarding the two ASD phenomena Multiple Scenario's and No Parallel Processes, precisely the phenomena that were not associated with social functioning as measured with the SCORS. Finally, interrater reliability was good to excellent. Taken together, the association of the ASD phenomena and the SCORS social cognition variables in the expected direction tentatively suggest that the ASD phenomena as they have been scored in the present study did tap low social cognition.

Second, although the patients with ASD were assessed by specialized clinicians, we did not confirm the diagnoses. Third, only a small number of ASD patients participated in the study, which might have affected the results. Fourth, the study sample is not fully representative of the actual population of people with ASDs. Another limitation of the study could be that a somewhat different set of cards was used for the control group and the ASD group. Whereas the control group described stories for the cards $1,2,13 \mathrm{MF}, 4,3 \mathrm{BM}$ and $7 \mathrm{GF}$, the ASD group saw the cards 1, 2, 5, $6 \mathrm{BM}, 13 \mathrm{MF}$ and 18BM. Although the content of the narrative is not relevant in scoring for ASD phenomena, the cards may have differed in the amount of details, emotional impact and the sort of relationship depicted. It cannot be entirely excluded that at least part of the differences between the two groups might be attributed to the differences in cards presented.

Therefore, even though results of this study are promising, more research is definitively needed to verify and confirm the present results. Such studies should include a larger sample of patients with ASD. In addition scoring of the TAT narratives should be done blind to the group the patient belongs to and further studies should use the same set of cards for all groups under study.

\section{Conclusion}

Although the use of the TAT in the assessment of patients who are suspected for an Autism Spectrum Disorder is rather unconventional and novel in clinical practice, the results of this preliminary study suggest the usefulness of a formal analysis of TAT narratives in the assessment process among adult patients who are suspected for Autism.

TAT narratives of patients with Autism are particularly characterized by weak central coherence, difficulties in describing and interpreting movements, and jumping to conclusions. These information processing problems may underlie the difficulties these patients may encounter in social interactions.

\section{References}

APA (2000). Diagnostic and statistical manual of psychiatric disorders IV-TR. Washington DC: American Psychiatric Association.

Ackerman, S. J., Hilsenroth, M. J., Clemence, A. J., Weatherill, R., \& Fowler, J. C. (2001). Convergent validity of Rorschach and TAT scales of object relations. Journal of Personality Assesment, 77, 295-306. doi:10.1207/S15327752JPA7702 11

Baron-Cohen, S. (2000) Theory of mind and autism: A fifteen year review. In S. Baron-Cohen, H. Tager-Flusberg, \& D. J. Cohen (Eds.), Understanding other minds. Perspectives from developmental cognitive neuroscience (pp. 3-21). Oxford: University Press.

Beaumont, R., \& Newcombe, P. (2006). Theory of mind and central coherence in adults with High-Functioning Autism or Asperger Syndrome. Autism, 10, 365-382. doi:10.1177/1362361306064416

Cicchetti, D. V. (1994). Guidelines, criteria, and rules of thumb for evaluating normed and standardized assessment instruments in psychology. Psychological Assessment, 6, 284-290. doi:10.1037/1040-3590.6.4.284

Cohen, J. (1977). Statistical power analysis for the behavioural sciences. New York: Academic Press.

Conklin, A., \& Westen, D. (2001). Thematic apperception test. In W. Dorfman, \& M. Hersen (Eds.), Understanding psychological assessment: Perspectives on individual differences (pp. 107-133). New York: Kluwer Academic/Plenum.

Cramer, P. (1999). Future directions for the Thematic Apperception Test. Journal of Personality Assessment, 72, 74-92. doi:10.1207/s15327752jpa7201_5

Edrisi, M., \& Eurelings-Bontekoe, E. H. M. (2009). Gifted, but hampered; early detection of Autism Spectrum Disorders among adults by means of clinical interview, personality questionnaires and the Thematic Apperception Test. Wetenschappelijk Tijdschrift Autisme, 2, 44-53.

Edrisi, M., \& Eurelings-Bontekoe, E. H. M. (2011). Doubts about the diagnosis. In R. Borra, R. van Dijk and R. Verboom (Eds.), Cultuur en psychodiagnostiek (pp. 185-197). Houten: Bohn Stafleu van Loghum. doi:10.1007/978-90-313-8580-5 13

Eurelings-Bontekoe, E. H. M., Luyten, P., \& Snellen, W. M. (2009). Validation of the theory driven profile interpretation of the Dutch Short form of the MMPI using the TAT Social Cognition and Object Relations Scale (SCORS). Journal of Personality Assessment, 91, 
155-165. doi:10.1080/00223890802634274

Happé, F., \& Frith, U. (2006). The weak coherence account: Detail-focused cognitive style in autism spectrum disorders. Journal of Autism and Developmental Disorders, 36, 5-25.

doi:10.1007/s10803-005-0039-0

Holaday, M., Moak, J., \& Shipley, M. A. (2001). Rorschach protocols from children and adolescents with Asperger's Disorder. Journal of Personality Assessment, 76, 482-495. doi:10.1207/S15327752JPA7603 09

Huprich, S. K., \& Greenberg, R. P. (2003). Advances in the assessment of object relations in the 1990s. Clinical Psychology Review, 23, 665-698. doi:10.1016/S0272-7358(03)00072-2

Langan-Fox, J., \& Grant, S. (2006). The Thematic Apperception Test: Toward a standard measure of the big three motives. Journal of Personality Assessment, 87, 277-291.

doi:10.1207/s15327752jpa8703 09

Minshew, N., Sweeney, J., \& Luna, B. (2002). Autism as a selective disorder of complex information processing and underdevelopment of neocortical systems. Molecular Psychiatry, 7, 14-15. doi:10.1038/sj.mp.4001166

Murray, H. (1943). Manual for the Thematic Apperception Test. Cambridge, MA: Harvard University Press.

Ozonoff, S., South, M., \& Provencal, S. (2005). Executive functions. In. F. R. Volkmar, R. Paul, A. Klin, \& D. Cohen (Eds.), Handbook of autism and pervasive developmental disorders (pp. 606-627). New Jersey: John Wiley and Sons.
Peters, E. J., Hilsenroth, M. J., Eudell-Simmons, E. M., Blagys, M. D., $\&$ Handler, L. (2006). Reliability and validity of the Social Cognition and Object Relations Scale in clinical use. Psychotherapy Research, 16, 617-626. doi:10.1080/10503300600591288

Porcerelli, J. H., Shahar, G., Blatt, S. J., Ford, R. Q., Mezza, J. A., \& Greenlee, L. M. (2006). Social cognition and object relations scale: Convergent validity and changes following inpatient treatment. Personality and Individual Differences, 41, 407-417.

Shrout, P. E., \& Fleiss, J. L. (1979). Intraclass correlations-uses in assessing rater reliability. Psychological Bulletin, 86, 420-428. doi:10.1037/0033-2909.86.2.420

Tager-Flusberg, H. (2007). Evaluating the Theory-of-Mind Hypothesis of Autism. Current Directions in Psychological Science, 16, 311-315. doi:10.1111/j.1467-8721.2007.00527.x

Tager-Flusberg, H., \& Caronna, E. (2007) Language disorders: au- tism and other pervasive developmental disorders. Pediatric Clinics of North America, 54, 469-481. doi:10.1016/j.pcl.2007.02.011

Westen, D. (1991a). Clinical assessment of object relations using the TAT. Journal of Personality Assessment, 56, 56-74. doi:10.1207/s15327752jpa5601_6

Westen, D. (1991b). Social cognition and object relations. Psychological Bulletin, 109, 429-455. doi:10.1037/0033-2909.109.3.429

Westen, D. (1985). Social Cognition and Object Relations Scale. Original manual to use for training. Ann Arbor: Department of Psychology, University of Michigan. 